\title{
Absence of jump discontinuity in the magnetization in quasi-one-dimensional random-field Ising models
}

\author{
Sanjib Sabhapandit \\ Department of Physics, University of Wisconsin, Madison, Wisconsin 53706, USA
}

(Published in: Phys. Rev. B 70, 224401 (2004))

\begin{abstract}
We consider the zero-temperature random-field Ising model in the presence of an external field, on ladders and in one dimension with finite range interactions, for unbounded continuous distributions of random fields, and show that there is no jump discontinuity in the magnetizations for any quasi-one dimensional model. We show that the evolution of the system at an external field can be described by a stochastic matrix and the magnetization can be obtained using the eigenvector of the matrix corresponding to the eigenvalue one, which is continuous and differentiable function of the external field.
\end{abstract}

PACS numbers: 75.10.Nr, 75.60.Ej

The nonequilibrium random field Ising model (RFIM) was proposed by Sethna et al. $\stackrel{1}{n}$ as a model for hysteresis and Barkhausen noise in ferromagnets. Since then, there has been a considerable theoretical interest in the nonequilibrium response of the model 1.2.3.4.5.6.7.8 Sethna et al. showed that in the mean-field limit, if the strength $\sigma$ of the quenched random field is large, the average magnetization per site is a continuous function of the external field, but for small $\sigma$, it shows a discontinuous jump as the external field is increased. However, in the mean-field limit, there is no hysteresis above the critical disorder $\sigma_{c}$, i.e., the magnetization follows the same curve in the increasing and decreasing field. This shortcoming of the mean-field limit can be overcome by working on a Bethe lattice, where the the model is solved exactly for the hysteresis ${ }^{2}$ and avalanche size distribution. ${ }^{3}$ Interestingly, the behavior of hysteresis loops on a Bethe lattice depends nontrivially on the coordination number $z$, so long as the distributions of the random fields are unbounded continuous. For $z=3$ the hysteresis loops show no jump discontinuity of magnetization even in the limit of small disorder, but for higher $z$ they do. This $z$ dependence of the hysteresis persists even for euclidean lattices. 4 The natural question to ask is to whether the existence jump discontinuity in magnetizations at low disorder depend only on the coordination number or also on the dimensionality of the lattice. In this paper we answer this question by showing the nonexistence of jump discontinuity in magnetizations for any quasi-one-dimensional RFIM, irrespective of the coordination number, for unbounded continuous distributions of random fields.

We first consider a two-leg ladder (shown in Fig. 1 of length $N$. At each vertex there is a Ising spin $s_{i}= \pm 1$ which interacts with nearest neighbors through a ferromagnetic interaction $J$, and coupled to the on-site quenched random field $h_{i}$ and the homogeneous external field $h$. The random fields $\left\{h_{i}\right\}$ are drawn independently from a unbounded continuous distribution $\phi\left(h_{i}\right)$. The Hamiltonian of the system is

$$
H=-J \sum_{<i, j>} s_{i} s_{j}-\sum_{i} h_{i} s_{i}-h \sum_{i} s_{i} .
$$

The system evolves under the zero-temperature Glauber single-spin-flip dynamics ${ }^{9}$ a spin flip is allowed only if it lowers the energy. We assume that the rate of spin flips is much larger than the rate at which $h$ is changed, so that all flippable spins may be said to relax instantly, and any spin $s_{i}$ always remains parallel to the net local field $\ell_{i}$ at the vertex

$$
s_{i}=\operatorname{sign}\left(\ell_{i}\right)=\operatorname{sign}\left(J \sum_{j=1}^{z} s_{j}+h_{i}+h\right) .
$$

We start with $h=-\infty$, when all the spins are down, and slowly increase the field. We are interested in the average magnetization of the system as a function of external field, away from the two ends of the ladder in the thermodynamic limit of $N \rightarrow \infty$.

Under the single spin-flip dynamics, for ferromagnetic coupling $(J>0)$, the system exhibits return point memory: if we start with with the state where all spins are down (at $h=-\infty)$, then state of the system at an increased field $h(T)$ at a later time $T$, does not depends on the detailed time dependent of the external field $h(t)$, and is same for all histories as long as the condition $h(t) \leq h(T)$ for all earlier times is obeyed. $\frac{1}{n}$ Therefore, to find the magnetization at $h$, we start with $h=-\infty$, so that all spins in the system are down and then increase the external field to $h$ in a single step. At that field several spins would become unstable. But for $J>0$, if we start with any stable configuration, and then increase the external field and allow the system to relax, the final stable configuration reached is independent of the order in which the unstable spins are flipped, as flipping of a spin can only increase the local field at its neighboring vertices and also in the relaxation process no spin flips more than once. Because of this abelian property of the spin flip, when the external field is increased from $-\infty$ to $h$ in a single step, we may choose to relax the spins from the two ends of the ladder as follows: We first relax the spins at the boundary ends (level 1). Then we

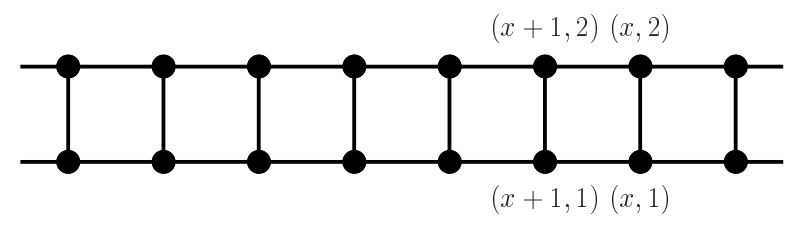

FIG. 1: Two-leg ladder 
relax spins next to the boundaries (at level 2) and so on with increasing levels as we move towards the center of the ladder. In the relaxation process, if a spin at level $x$ flips up, we check all the spins at lower levels for possible upward flips, before flipping the second spin at level $x$. Note that the state of the spins at a level $r$ from the right end is independent of the state at level $l$ from the left end, so long as all the spins in between levels are kept down. Therefore we can relax the spins from the two boundary ends on the left and right halves of the ladder independently (which are identical).

We choose our coordinates on each halves of the ladder such that the level $x$ consists of vertices $(x, 1)$ and $(x, 2)$. Let $P_{h}^{x}\left(s_{1}, s_{2}\right)$ be the conditional probability that, in the earlier spin relaxation scheme, the spins at level $x$ reach a final state $\left\{s_{1}, s_{2}\right\}$, i.e., the spin at the vertex $(x, 1) \rightarrow s_{1}$ and the spin at the vertex $(x, 2) \rightarrow s_{2}$; given that the spins at level $x+1$ are kept down and all the spins at the lower levels are relaxed and the external field is $h$. Corresponding to four possible final states we get four probabilities $P_{h}^{x}(1,1)$, $P_{h}^{x}(1,-1), P_{h}^{x}(-1,1)$ and $P_{h}^{x}(-1,-1)$, which depend on the states at level $x-1$ and the random fields at level $x$. Note that $P_{h}^{x}(1,-1)=P_{h}^{x}(-1,1)$, due to the symmetry of the lattice.

To illustrate how to obtain the recursions for the probabilities $P_{h}^{x}\left(s_{1}, s_{2}\right)$ 's, we consider an example where the final $\{1,1\}$ state at level $x$ is achieved from its initial state $\{-1,-1\}$, when state at level $x-1$ is $\{1,1\}$. We denote $p_{m}(h)$ with $0 \leq m \leq 3$ as the conditional probability that the local field at any vertex $i$ will be large enough so that it will flip up, if $m$ of its neighbors are up, when the uniform external field is $h$. Clearly, for a given distribution of random fields $\phi\left(h_{i}\right)$ :

$$
p_{m}(h)=\int_{(3-2 m) J-h}^{\infty} \phi\left(h_{i}\right) d h_{i}, \quad 0 \leq m \leq 3 .
$$

Since the spin at vertex $(x, 1)$ has one up neighbor, it will flip up with probability $p_{1}(h)$. Now the spin at vertex $(x, 2)$ has two up neighbors, so it flips up with probability $p_{2}(h)$. But the local field at vertex $(x, 1)$ may not be large enough for the spin to flip up when it has only one up neighbor. In this case, the spin at vertex $(x, 2)$ flips up first, with probability $p_{1}(h)$, and then the spin at vertex $(x, 1)$ flips up, when is has two up neighbors, with probability $\left[p_{2}(h)-p_{1}(h)\right]$. Therefore, the total probability of the spins at level $x$ flipping up via this process is $P_{h}^{(x-1)}(1,1)\left\{p_{1}(h) p_{2}(h)+\left[p_{2}(h)-p_{1}(h)\right] p_{1}(h)\right\}$.

The case where the state at level $x-1$ is $\{-1,-1\}$, the flipping of the spin at vertex $(x, 1)$ might causes the spin at vertex $(x-1,1)$ to flip up and as a result the spin at vertex $(x-1,2)$ might flip up, while the spin at vertex $(x, 2)$ is still kept down. We denote the probability of this spin flip process by $Q_{h}^{(x-1)}$. If we consider $\left[P_{h}^{x}(1,1), 2 P_{h}^{x}(-1,1), P_{h}^{x}(-1,-1)-Q_{h}^{x}, Q_{h}^{x}\right]$ as a column vector $\mathcal{P}_{x}(h)$, then the recursion relations for these probabilities can be represented in matrix form

$$
\mathcal{P}_{x}(h)=W_{h} \mathcal{P}_{x-1}(h),
$$

where $W_{h}$ is a $4 \times 4$ matrix whose elements $w_{i j}$ 's are polynomials in $p_{m}(h)$ 's.

For a given probability distribution of random fields $\phi\left(h_{i}\right)$ and value of external field $h$, we determine $p_{m}(h)$, and then, using Eq. (4) we can recursively determine the probabilities represented by the column vector $\mathcal{P}_{x}(h)$. Note that the matrix $W_{h}$ is column stochastic, ${ }^{10}$ i.e., $w_{i j} \geq 0$ and $\sum_{i=1}^{4} w_{i j}=1$. Therefore, for large $x$, the vector $\mathcal{P}_{x}(h)$ tends to a limiting vector $\mathcal{P}^{\star}(h)$, which is the eigenvector of the matrix $W_{h}$, corresponding to the eigenvalue one (the maximal eigenvalue). The variation of $\mathcal{P}^{\star}(h)$ with respect to the external field $h$ is ${ }^{11}$

$$
\frac{d \mathcal{P}^{\star}(h)}{d h}=A_{h}^{\#} \frac{d W_{h}}{d h} \mathcal{P}^{\star}(h),
$$

where $A_{h}^{\#}$ is the group inverse of the matrix $A_{h}=I-W_{h}$, letting $I$ denote identity matrix. The entries of $A_{h}^{\#}$ are continuous functions of $h$ for continuous distributions of random fields. Since according to Eq. 3 the elements of $W_{h}$ are continuous and differentiable (with continuous first derivative), $\mathcal{P}^{\star}(h)$ is a continuous and differentiable (with continuous first derivative) function of $h$.

To calculate the magnetization at the center vertex $(O, 1)$, in the limit $N \rightarrow \infty$, we keep the spins at level $O$ down and relax the full system. The states of the spins at the adjacent levels on both sides are given independently by the probability vector $\mathcal{P}^{\star}(h)$. Now we relax the spins at at level $O$. Let $P_{h}^{O}(-1,-1)$ be the probability that in this relaxation process the spins at level $O$ do not flip up and $P_{h}^{O}(-1,1)$ be the probability that only the spin at vertex $(O, 2)$ flips up. The magnetization at the vertex $(O, 1)$ is

$$
M_{O}(h)=1-2\left[P_{h}^{O}(-1,-1)+P_{h}^{O}(-1,1)\right] .
$$

In the limit $N \rightarrow \infty$, all the vertices deep inside the ladder are equivalent. Therefore, $M_{O}(h)$ gives the average magnetization on the ladder far away from the boundary. Since for continuous distributions of random fields $\mathcal{P}^{\star}(h)$ is continuous and differentiable, the average magnetization is also continuous and differentiable function of $h$.

We have compared the theoretical calculation with numerical simulation results. The points in Fig. 2 show the results of simulations for a single realization of quenched Gaussian random fields with mean 0 and standard deviation $\sigma=0.5 \mathrm{~J}$ and $\sigma=1.5 \mathrm{~J}$, respectively. We use periodic boundary condition along the length of a ladder with $N=2^{23}$. Different runs using different realizations of quenched field give results which are indistinguishable at the scale of the graph. The solid curves the Fig. 2]are obtained using theoretical calculation.

The analysis of a two-leg ladder can be extended to a case of an $n$-leg ladder (with finite $n$ ), where the relaxation scheme is such that, all the $n$ spins at the same level $x$ are relaxed before relaxing the spins at level $x+1$. The relaxation process can be represented by the probabilities $P_{h}^{x}\left(s_{1}, s_{2}, \ldots, s_{n}\right)$ of the states of spins at level $x$ and the probabilities $Q_{i}^{x}(h)$ 's for intermediate relaxations. Now these probabilities at level $x$ can be obtained from the probabilities at level $x-1$, and using proper linear combinations these can be expressed by a matrix recursion relation like Eq. (4). It is simpler to consider the $n$-leg ladder to wrapped around a cylinder (of course the resultant magnetization is quantitatively different from the case of an $n$-leg ladder, but the qualitative behavior remains unchanged), since then many of the probabilities become equal, 


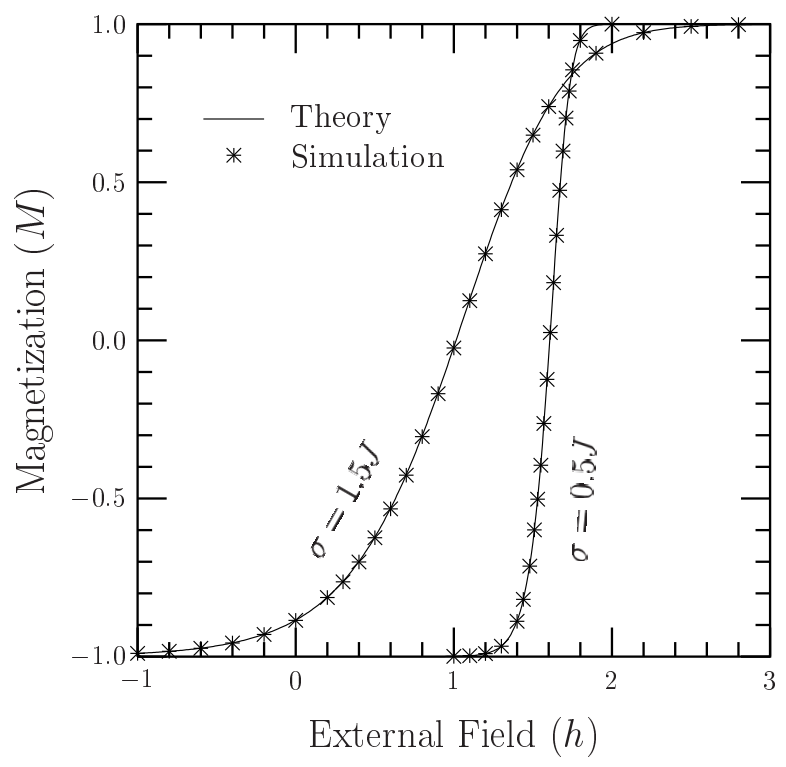

FIG. 2: Magnetization curves for a two-ladder in the increasing field. $N=2^{23}$.

due to the rotational symmetry of the cylinder. As an example, consider the case $n=3$. The state at a level $x$, when the spins at level $x+1$ are kept down, can be represented by only using $P_{h}^{x}(1,1,1), P_{h}^{x}(1,1,-1), P_{h}^{x}(1,-1,-1)$, and $P_{h}^{x}(-1,-1,-1)$. The $Q_{i}(h)$ 's needed to describe the relaxation at level $x-1$, when relaxing the spins at level $x$ are obtained as follows:

(a) Suppose the state at level $x-1$ is $\{1,-1,-1\}$ and the spin at vertex $(x, 2)$ flips up and the spin at vertex $(x, 3)$ is kept down. We define $Q_{1}^{(x-1)}(h)$ to be the probability that the spin at vertex $(x-1,2)$ flips up and subsequently the spin at vertex $(x-1,3)$ flips up as a result. (b) Suppose the state at level $x-1$ is $\{-1,-1,-1\}$ and the spin at vertex $(x, 1)$ flips up while the spins at vertices $(x, 2)$ and $(x, 3)$ are kept down. Now we define: (i) $Q_{2}^{(x-1)}(h)$, the probability that the spin at vertex $(x-1,1)$ flips up and as a result the spins at vertices $(x-1,2)$ and $(x-1,3)$ flip up. (ii) $Q_{3}^{(x-1)}(h)$, the probability that the spin at vertex $(x-1,1)$ flips up and as a result the spin at vertices $(x-1,2)$ flips up but the spin at vertex $(x-1,3)$ remains down. (iii) $Q_{4}^{(x-1)}(h)$, the probability that the spin at vertex $(x-1,1)$ flips up but the spins at vertices $(x-1,2)$ and $(x-1,3)$ remain down, and (iv) $Q_{5}^{(x-1)}$, the probability that the spins at vertices $(x-1,2)$ and $(x-1,3)$ flip up after we flip the spin at vertex $(x, 2)$ and the spin at vertex $(x, 3)$ is still kept down.

Now the elements of the probability vector $\mathcal{P}_{x}(h)$ are $P_{h}^{x}(1,1,1), 3 P_{h}^{x}(1,1,-1), 3\left[P_{h}^{x}(1,-1,-1)-Q_{1}^{x}(h)\right]$, $3 Q_{1}^{x}(h), P_{h}^{x}(-1,-1,-1)-Q_{2}^{x}(h)-Q_{3}^{x}(h)-Q_{4}^{x}(h), Q_{2}^{x}(h)$, $Q_{3}^{x}(h), Q_{4}^{x}(h)-Q_{5}^{x}(h)$ and $Q_{5}^{x}(h)$. The recursion relation for $\mathcal{P}_{x}(h)$ is represented by Eq. 4 with a $9 \times 9$ stochastic matrix $W_{h}$ whose elements can be expressed in terms of $p_{m}(h)$ 's. Finally, the magnetization can be obtained using the limiting vector $\mathcal{P}^{\star}(h)$ and $p_{m}(h)$ 's.

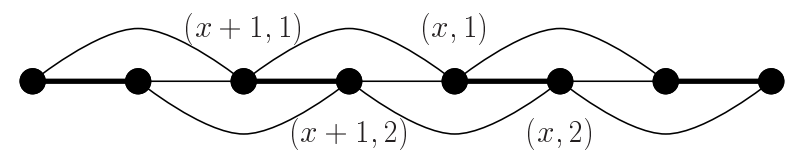

(a)

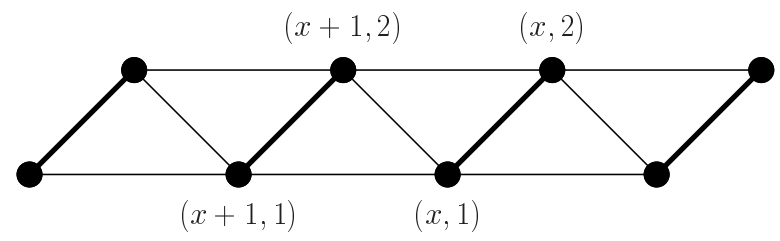

(b)

FIG. 3: (a) Linear chain showing nearest and next neighbor interactions and (b) graph on which nearest neighbor interaction is equivalent to the linear chain with nearest and next neighbor interactions.

We next consider the RFIM in one dimension (1D), with "finite range interactions" - each spin interacts with all the spins up to a distance $n$, through ferromagnetic interaction $J$. We take the length of the chain $N$ (later we will take the limit $N \rightarrow \infty$ ) to be a multiple of $n$. Now we can group $n$ spins together in a single level and relax the spins from the two boundaries such that we relax all the spins at the lower levels before relaxing the spins at an higher level.

As a concrete example, we calculate the magnetization for $n=2$, i.e., each spin interacts with the nearest and next nearest neighbors. The interactions are shown in Fig. 3]a). This model is also equivalent to the RFIM with nearest neighbor interaction on the graph shown in Fig. 3 b). Let $P_{h}^{x}\left(s_{1}, s_{2}\right)$ be the conditional probability that the spins at level $x$ reach a final state $\left\{s_{1}, s_{2}\right\}$, i.e., the spin at the vertex $(x, 1) \rightarrow s_{1}$ and the spin at the vertex $(x, 2) \rightarrow s_{2}$; given that the spins at level $x+1$ are kept down and all the spins at the lower levels are relaxed. We need to define four more probabilities for the further relaxation at level $x-1$, as a result of a spin flip at level $x$.

Consider the following cases of relaxing the spins at level $x$ from its original state $\{-1,-1\}$ : (a) Suppose the state at level $x-1$ is $\{-1,1\}$ and the spin at vertex $(x, 2)$ flips up, given that spins at vertex $(x, 1)$ and level $x+1$ are kept down. It may cause the spin at vertex $(x-1,1)$ to flip up and we denote the probability of it to flip up by $Q_{1}^{x-1}(h)$. The spin at vertex $(x-$ $1,1)$ remains down with probability $P_{h}^{x-1}(-1,1)-Q_{1}^{x-1}(h)$. (b) Suppose the state at level $x-1$ is $\{-1,-1\}$ and the spin at vertex $(x, 2)$ flips up, given that spins at vertex $(x, 1)$ and level $x+1$ are kept down. This may finally cause the spin at vertex $(x-1,1)$ to flip up. The spin at vertex $(x-1,2)$ may or may not flip up during the relaxation. We denote the probability of the spin at vertex $(x-1,1)$ flipping up by $Q_{2}^{x-1}(h)$. It remains down with probability $P_{h}^{x-1}(-1,-1)-Q_{2}^{x-1}(h)$. (c) Suppose the state at level $x-1$ is $\{-1,-1\}$ and the spin at 


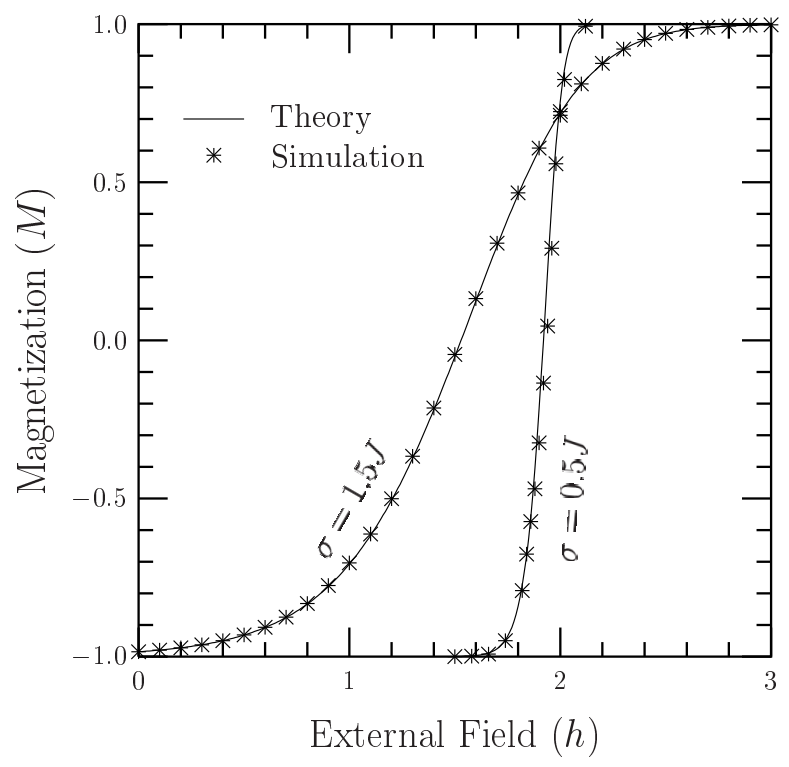

FIG. 4: Magnetization curve in the increasing field for linear chain with nearest and next neighbor interactions. $N=2^{23}$.

vertex $(x, 1)$ flips up, given that spins at vertex $(x, 2)$ and level $x+1$ are kept down. Now we relax the spins at level $x-1$. The spin at vertex $(x-1,2)$ can not flip up in this further relaxation unless the spin at vertex $(x-1,1)$ flips up first. Let $Q_{3}^{x-1}(h)$ be the probability that the spin at vertex $(x-1,1)$ flips up, but it can not cause the spin at vertex $(x-1,2)$ to flip up. We denote the probability of both the spins at level $x-1$ flipping up by $Q_{4}^{x-1}(h)$. Therefore, the spins at level $x-1$ remain down with probability $P_{h}^{x-1}(-1,-1)-Q_{3}^{x-1}(h)-Q_{4}^{x-1}(h)$.

We consider the probabilities $\left[P_{h}^{x}(1,1), P_{h}^{x}(1,-1)\right.$, $P_{h}^{x}(-1,1)-Q_{1}^{x}(h), P_{h}^{x}(-1,-1)-Q_{2}^{x}(h), Q_{1}^{x}(h), Q_{2}^{x}(h)-$
$\left.Q_{3}^{x}(h)-Q_{4}^{x}(h), Q_{3}^{x}(h), Q_{4}^{x}(h)\right]$ as a column vector $\mathcal{P}_{x}(h)$, so that the recursion relations of the probabilities can be represented in matrix form as in Eq. 4 with a $8 \times 8$ stochastic matrix $W_{h}$. We find the eigenvector $\mathcal{P}^{\star}(h)$ for the matrix $W_{h}$ and using $\mathcal{P}^{\star}(h)$ and $p_{m}(h)$ 's calculate the average magnetization far away from the boundary. Figure 4 shows the comparison between theoretical and simulation results for Gaussian quenched random fields with $\sigma=0.5 \mathrm{~J}$ and $\sigma=1.5 \mathrm{~J}$. The simulation results are obtained for single realizations, on a linear chain of length $N=2^{23}$ with nearest and next neighbor interactions, with periodic boundary condition.

In summary, we have demonstrated that there is no jump discontinuity in the magnetizations for any quasi-one dimensional RFIM for unbounded continuous distributions of random fields. We showed that for the RFIM on an $n$-leg ladder and in $1 \mathrm{D}$ with interactions extended to $n$ closest neighbors, for finite $n$ the relaxation of spins at an external field $h$ can be described by a stochastic matrix $W_{h}$; and the average magnetization can be obtained using the eigenvector of $W_{h}$ corresponding to the eigenvalue one, which is a continuous and differentiable function of $h$, for unbounded continuous distributions of random fields. We explicitly calculated the magnetizations for two simpler cases: (a) the two-leg ladder and (b) in 1D with nearest and next nearest neighbors interactions; and confirmed our results using numerical simulation for Gaussian distribution of random fields. The question of how to take the $n \rightarrow \infty$ limit, where the magnetizations show jump discontinuity below a critical disorder as the external field is varied, remains open.

The author would like to thank D. Dhar and S. N. Coppersmith for their useful comments on the manuscript. This work was supported by National Science Foundation grant No. DMR-0209630.
1 J. P. Sethna, K. A. Dahmen, S. Kartha, J. A. Krumhansl, B. W. Roberts, and J. D. Shore, Phys. Rev. Lett. 70, 3347 (1993).

2 D. Dhar, P. Shukla, and J. P. Sethna, J Phys A 30, 5259 (1997).

3 S. Sabhapandit, P. Shukla, and D. Dhar, J. Stat. Phys. 98, 103 (2000).

4 S. Sabhapandit, D. Dhar, and P. Shukla, Phys. Rev. Lett. 88, 197202 (2002).

5 P. Shukla, Phys. Rev. E 62, 4725 (2000); 63, 027102 (2001).

${ }^{6}$ F. Colaiori, A. Gabrielli, and S. Zapperi, Phys. Rev. B 65, 224404 (2002).

7 R. Dobrin, J. H. Meinke, and P. M. Duxbury, J Phys A 35, L247 (2002).
${ }^{8}$ P. M. Bleher, J. Ruiz, and V. A. Zagrebnov, J. Stat. Phys. 93, 33 (1998), and references therein.

${ }^{9}$ See, for example: K. Kawasaki, Phase Transitions and Critical Phenomena, edited by C. Domb and M. S. Green (Academic Press, London, 1972), vol 2.

10 A. Berman and R. J. Plemmons, Nonnegative Matrices in the Mathematical Sciences (Academic Press, New York, 1979).

11 G. H. Golub and C. D. Meyer, SIAM J. Algebraic Discrete Methods 7, 273 (1986); C. D. Meyer, SIAM J. Matrix Anal. Appl. 15, 715 (1994) 